\title{
Potencial de la investigación "En relevo" para la construcción de saberes y la producción científica
}

\section{Potential of research "In relief" for the construction of knowledge and scientific production}

\author{
Javier García Reynaud*
}

ccnn@upnfm.edu.hn

\section{Resumen}

Con la finalidad de evaluar su potencial para la generación de aprendizaje significativo y conocimiento científico, se exploraron y describieron los resultados de la implementación piloto de una metodología de investigación alternativa denominada "en relevo", en la cual 8 equipos de estudiantes de 8 secciones del espacio formativo "Introducción a la Educación Ambiental" participaron secuencial y transitoriamente en la recolección y análisis de datos hasta la presentación de resultados de investigación. Se evaluó la calidad de los reportes parciales generados por los equipos en un proyecto de investigación llevado a cabo desde abril de 2014 a agosto de 2015 en la temática de calidad de aguas superficiales y se analizó la prevalencia de procesos generadores de aprendizaje significativo en la participación de cada uno. Se encontró que en procedimientos tales como la selección de literatura, calibración y pilotaje de instrumentos y aplicación de normativas básicas de citación y referencias, los cuales presentaron valores de calidad inferiores al mínimo aceptable, es fundamental el adiestramiento previo y el acompañamiento por parte de la figura de coordinación. Asimismo, etapas como la construcción del sustento teórico y metodológico de la investigación piloto mostraron una prevalencia significativamente superior de procesos potenciadores de aprendizaje significativo que aquella evidenciada en etapas tales como la construcción de contexto y la recolección y análisis de información de campo.

" Universidad Pedagógica Nacional "Francisco Morazán", Departamento de Ciencias Naturales. Recibido 16 de marzo de 2017 /Aceptado 30 de mayo de 2017. la Universidad Pedagógica Nacional Francisco Morazán, de Honduras, se comparten bajo términos de la Licencia Creative Commons: Se permite que otros puedan descargar las obras y compartirlas con otras personas, siempre y cuando se reconozca su autoria, pero no se pueden cambiar de ninguna manera ni se pueden utilizer comercialmente. 
Palabras clave: investigación en relevo, aprendizaje significativo, indagación científica, investigación secuencial.

\section{Abstract}

In order to evaluate its meaningful learning and scientific knowledge generating potential, the results of a pilot implementation of an alternative research methodology called "in relay", were explored and described. 8 teams of 8 students of the course "Introduction to Environmental Education" participated sequentially and transiently from collecting and analyzing data to presenting research results. Quality of partial reports generated by students participating in a research project, conducted from April 2014 to August 2015 on the subject of superficial water quality, and prevalence of meaningful learning generating processes was evaluated. It was found that in processes such as selection of literature, calibration and piloting of tools, and application of basic rules of citation and referencing, which presented quality values below the minimum acceptable, prior training and accompaniment by the lead researcher is essential. In addition, stages such as the construction of the research theoretical and methodological framework showed a significantly higher prevalence of meaningful learning generating processes than that evidenced in stages such as the construction of context and the collection and analysis of field data.

Keywords: relay research; meaningful learning; scientific inquiry; sequential research.

\section{Introducción}

Los proyectos de investigación científica con la participación activa de estudiantes en los centros de educación superior en Honduras presentan la característica recurrente de llevarse a cabo como requerimientos de fin de carrera y como productos puntuales en un modesto número de espacios formativos específicamente orientados al desarrollo de competencias investigativas, más que como actividades transversales en los planes de estudio.

Tal situación, además de impactar en el número de

<Paradigma> - Revista de Investigación Educativa. Año 24. No. 37 
proyectos de investigación desarrollados desde las universidades, que muestran valores relativamente bajos en el área de las Ciencias Naturales (Universidad Pedagógica Nacional Francisco Morazán, 2011), limita la experiencia que podría acumularse en el estudiantado al verse involucrado en diferentes etapas y temáticas de investigación a lo largo de su formación y recorta la diversidad de estrategias para la construcción de aprendizajes en el nivel superior en Honduras.

Sin desconocer el papel que factores económicos, humanos y técnicos representan para el agravamiento del problema, la relativamente limitada generación de conocimiento a través de la investigación científica a nivel universitario está también relacionada en parte a la duración trimestral de los períodos académicos, normalmente incompatible con el tiempo necesario para llevar a cabo un proceso de investigación científica formal de principio a fin desde los espacios formativos.

Se exploró-describió el ensayo piloto de una metodología denominada "en relevo", en la cual se desarrolla un proyecto de investigación desde el planteamiento del problema hasta la generación de resultados desde un solo espacio formativo a lo largo de varios períodos académicos, lo cual involucra a más de un equipo de estudiantes que toman secuencialmente los avances del equipo anterior, hasta generar respuestas a las preguntas de investigación originalmente definidas con la finalidad de permitir la generación de aprendizaje tanto a nivel de contenido del tema de estudio como de las etapas de los procesos de investigación en el área de las Ciencias Naturales. la Universidad Pedagógica Nacional Francisco Morazán, de Honduras, se comparten bajo términos de la Licencia Creative Commons: Se permite que otros puedan descargar las obras y compartirlas con otras personas, siempre y
cuando se reconozca su autoría, pero no se pueden cambiar de ninguna manera ni se pueden utilizer comercialmente. 


\section{Discusión teórica}

Shavelson y Towne (2002), luego de un profundo análisis interdisciplinar de la filosofía de la ciencia, de investigaciones en las ciencias naturales y sociales y de la sistematización de información proporcionada por autoridades del financiamiento y administración de investigación educativa en los Estados Unidos proponen una serie de fundamentos a los que denominan "principios de la indagación científica", de los cuales afirman acentúan la objetividad, rigurosidad de pensamiento, apertura mental y honesta y rigurosa presentación de resultados. Tales principios se presentan a continuación:

1. Propone preguntas relevantes que pueden ser investigadas empíricamente.

2. Enlaza investigación con teoría relevante.

3. Utiliza métodos que permiten la investigación directa de la preguntas de investigación.

4. Provee una cadena de razonamiento coherente y explícito.

5. Replica y generaliza entre estudios.

6. Revela la investigación realizada para estimular la crítica y el escrutinio profesional.

Los principios presentados constituyen, más que un algoritmo infalible para la indagación científica, un marco de referencia que indica cómo las inferencias de la investigación deben en general ser apoyadas o refutadas por un núcleo de procesos independientes, herramientas y prácticas.

Métodos alternativos de investigación como la investigación en relevo, teniendo en cuenta la necesidad de analizar los resultados de su implementación exploratoria, consideró entonces evaluar la calidad desde la perspectiva de tales principios de la indagación científica.

La puesta en práctica de una metodología de investigación "en relevo", por otro lado, vista como una alternativa para diversificar las estrategias para construir aprendizaje significativo, y considerando las ventajas implícitas de este tipo de aprendizaje en contraposición con el aprendizaje memorístico, documentadas por Alonso (2010) y Novak (2011), constituye también una motivación de elevada importancia para la implementación piloto de la metodología.

<Paradigma> - Revista de Investigación Educativa. Año 24. No. 37 
Para analizar las rutas a través de las cuales la generación de condiciones asociadas con el aprendizaje significativo a nivel individual puede resultar posible a partir de la investigación colectiva y en relevo, es relevante como punto de partida notar como Ausubel (1983) enfatiza en la diferencia entre el verdadero aprendizaje significativo y la simple conexión no sustantiva y arbitraria de información nueva con información previa. Lo anterior se vuelve relevante al considerar que la transformación y evolución del nuevo contenido y de la estructura existente es una condición absolutamente necesaria para lograr aprendizajes a largo plazo y verdaderamente sustantivos a partir de actividades potenciadoras.

Tal transformación se hará posible a través de tres mecanismos presentados por Ausubel (1983) como "tipos" de aprendizaje significativo: aprendizaje significativo representacional, aprendizaje significativo conceptual y aprendizaje significativo proposicional.

El primero de estos tipos, el aprendizaje representacional, es para Ausubel la forma más elemental de aprendizaje significativo; asimismo es del cual dependen los demás tipos de aprendizaje y consiste en atribuir significados a determinados símbolos. En palabras de Ausubel (1983), el aprendizaje representacional "ocurre cuando se igualan en significado símbolos arbitrarios con sus referentes (objetos, eventos, conceptos) y significan para el alumno cualquier significado al que sus referentes aludan".

Un segundo tipo de aprendizaje significativo, al cual se le atribuye un mayor estado de evolución con respecto al aprendizaje representacional por involucrar procesos de mayor complejidad y ser dependientes de este primer tipo, es el aprendizaje de conceptos. Para Ausubel, los conceptos son "objetos, eventos, situaciones o propiedades que poseen atributos criteriales (características) comunes y que se designan mediante algún símbolo o signos aceptados". Los conceptos, para Ausubel (2000), constituyen entonces un importante aspecto de asimilación de teoría considerando el hecho de que la comprensión y procesos como la resolución significativa de problemas dependen precisamente de la disponibilidad de éstos en la estructura cognitiva del que aprende. Es evidente que los seres humanos interpretan las experiencias "crudas" percibidas en términos de conceptos particulares existentes en sus estructuras cognitivas. En este sentido, los conceptos constituyen los ladrillos para la construcción de aprendizaje significativo la Universidad Pedagógica Nacional Francisco Morazán, de Honduras, se comparten bajo términos de la Licencia Creative Commons: Se permite que otros puedan descargar las obras y compartirlas con otras personas, siempre y
cuando se reconozca su autoría, pero no se pueden cambiar de ninguna manera ni se pueden utilizer comercialmente. 
y resolución de problemas. Un tercer y más complejo tipo de aprendizaje significativo, con marcadas vinculaciones al proceso de generación conocimiento a partir de la práctica investigativa en relevo es el aprendizaje proposicional.

La mayor complejidad atribuida por Ausubel al aprendizaje proposicional se explica por el hecho de que más allá de asimilar lo que por sí solas o en combinación representan los símbolos (las palabras), este tercer tipo de aprendizaje demanda la adquisición del significado de las ideas en forma de proposiciones. En este sentido, por proposiciones se hace referencia a ideas en la forma de declaraciones que poseen significado "denotativo", es decir, que evocan características al oír los conceptos y significado "connotativo", por llevar también consigo una carga emotiva, actitudinal o idiosincrática. En esencia, en el contexto de este tipo de aprendizaje, las proposiciones interactúan con las ideas relevantes ya establecidas en la estructura cognoscitiva del estudiante, siendo justamente de esta interacción de la cual surgen significados de nuevas proposiciones.

En este sentido, como uno de los objetivos fundamentales del presente estudio, identificar evidencias de procesos que han sido asociados a la construcción de aprendizaje significativo, tales como la definición de términos (aprendizaje significativo representacional) y la construcción y conexión de conceptos (aprendizaje significativo conceptual y proposicional, respectivamente) en el área de las ciencias, se vuelve una actividad relevante en el sentido de generar insumos para la evaluación de mecanismos alternativos generadores de aprendizaje.

\section{Métodos y materiales}

Población y Muestra

Se realizó un muestreo no probabilístico por conveniencia en una población constituida por los estudiantes matriculados en el período enero 2014 a octubre 2015 en jornada matutina del espacio formativo de Introducción a la Educación Ambiental, en la Universidad Pedagógica Nacional Francisco Morazán, de la cual se seleccionó como muestra a los estudiantes de la Carrera de Ciencias Naturales matriculados en el espacio, lo que representó a lo largo de 5 períodos académicos, un total de 31 estudiantes de 8 equipos de trabajo.

<Paradigma> - Revista de Investigación Educativa. Año 24. No. 37 


\section{Instrumentos}

\section{Determinación de la calidad del proceso investigativo "en relevo"}

Cada reporte parcial de investigación fue evaluado tomando como base el documento "Rúbrica para trabajos de investigación", diseñado, evaluado y corregido por el Comité de Avalúo del Aprendizaje Estudiantil, de la Escuela Graduada de Ciencias y Tecnologías de la Información (EGCTI), en la Universidad de Puerto Rico (2011), adaptándolo al contexto de la presente implementación piloto mediante la selección de aquellos indicadores que evaluaran los principios de indagación científica propuestos por Shavelson y Towne (2002), lo que resultó en el documento: "Rúbrica para la evaluación de reportes parciales de investigación".

Ocho dimensiones (Calidad en la definición del objeto de estudio; Calidad en la revisión de literatura; Calidad en las fuentes de información; Calidad en la definición de la metodología; Calidad en la recopilación de datos; Calidad en el análisis de datos; Calidad en las conclusiones del estudio y Calidad en términos de formato y estilo) fueron desagregadas en 18 indicadores que se evaluaron con la rúbrica.

Para reducir el sesgo en la evaluación de calidad, el instrumento fue aplicado adicionalmente por una docente de la Facultad de Ciencia y Tecnología independiente del estudio para cada uno de los informes de investigación parcial.

Análisis de la prevalencia de procesos potenciadores del aprendizaje significativo

Se contabilizaron y ponderaron los indicadores de procesos potenciadores de aprendizaje representacional, conceptual y proposicional en cada reporte parcial de investigación. Las definiciones de términos en cada reporte fueron considerados indicadores de procesos asociados a la construcción de aprendizaje representacional; la identificación y/o diferenciación de características o atributos comunes a objetos o categorías de objetos fueron consideradas indicadores de procesos asociados a la construcción de aprendizaje de conceptos y finalmente las conexiones de conceptos en la forma de demostraciones, relaciones o proposiciones presentadas de forma escrita en cada informe parcial de investigación fueron consideradas indicadores de procesos asociados a la construcción de aprendizaje proposicional. la Universidad Pedagógica Nacional Francisco Morazán, de Honduras, se comparten bajo términos de la Licencia Creative Commons: Se permite que otros puedan descargar las obras y compartirlas con otras personas, siempre y
cuando se reconozca su autoría, pero no se pueden cambiar de ninguna manera ni se pueden utilizer comercialmente. 
Se asignaron puntajes a los indicadores de procesos asociados al aprendizaje representacional, (1 punto por indicador identificado), aprendizaje conceptual ( 2 puntos por indicador identificado) y aprendizaje proposicional ( 4 puntos por indicador identificado). Para reducir el sesgo en la identificación de indicadores de procesos de aprendizaje representacional, conceptual y proposicional, además del criterio del investigador principal, se contó con la opinión de una docente de la facultad de Ciencia y Tecnología independiente al proceso de análisis de datos quien aprobó o desaprobó los indicadores (o "evidencias") identificados, y propuso nuevos que no habían sido señalados bajo el criterio del investigador principal.

\section{Procedimiento de recogida y análisis de datos}

Para evaluar el desempeño de la metodología de investigación "en relevo" fue definido un proyecto de investigación en el tema de calidad de aguas superficiales, seleccionado bajo el criterio de que debería encontrarse dentro del contenido previsto para el espacio formativo e involucrar la recolección de datos de campo mediante el uso de equipo disponible en los laboratorios del centro de estudios. El problema de estudio consistió en determinar la correlación entre nutrientes habituales de cuerpos de agua superficiales y la concentración de oxígeno disuelto en la quebrada Agua Salada del Distrito Central, Francisco Morazán, Honduras.

Un primer paso para todos los equipos participantes fue la revisión del contenido base del tema del estudio, facilitado por el docente a cargo del espacio. A partir del segundo equipo de investigación en relevo, en la etapa inicial fue también solicitada la revisión de los avances logrados por equipos antecesores documentados en sus informes parciales de investigación.

Como segundo paso en el desarrollo de las etapas de investigación se asignó a cada equipo la función de recolectar las muestras de agua en un punto seleccionado del recorrido de la quebrada Agua Salada, las cuales fueron analizadas in-situ para oxígeno disuelto y en laboratorio por medio de análisis de colorimetría para valores de nitrito, nitrato, amonio y fosfato.

Luego de medir y registrar los valores de los parámetros se asignó al equipo investigador la etapa que en función de la secuencia lógica y

<Paradigma> - Revista de Investigación Educativa. Año 24. No. 37 
los avances generados por el equipo previo resultara pertinente. En este sentido un equipo que recibiera como avances realizados por su antecesor un marco teórico finalizado, procedería a sentar las bases de un marco metodológico a ser continuado por un equipo posterior, para continuar con esa lógica hasta la generación de respuestas a las preguntas de investigación planteadas originalmente por el equipo primero meses antes.

\section{Análisis de datos}

La "Rúbrica para la evaluación de reportes parciales de investigación" contó para cada indicador de calidad con 4 posibles descriptores, los cuales presentan características desde la más deseable, pasando por dos niveles intermedios, hasta la menos deseable y que asignaron un puntaje en los rangos de 8-7; 6-5; 4-3 o 2-1 al compararlos con las características presentes en los reportes de investigación.

Se calculó la calificación total obtenida en cada reporte, el total de indicadores con calificación "N/A" (en el caso uno o varios de los indicadores no estuviera dentro de las funciones asignadas al equipo), el puntaje máximo posible excluyendo los indicadores "N/A" y la proporción del puntaje alcanzado (total del reporte e individual de cada dimensión de calidad) con respecto al puntaje máximo posible, la cual fue expresada en porcentaje.

Las calificaciones generadas por ambos revisores fueron com paradas con una prueba T de Student para medias de muestras emparejadas, con la finalidad de encontrar similitudes en el comportamiento del desempeño de los reportes.

Se utilizaron los parámetros de calidad vigentes en la UPNFM por lo que un valor de calidad de $65 \%$ fue tomado como valor mínimo satisfactorio.

\section{Prevalencia de procesos potenciadores del aprendizaje significativo}

La interpretación cuantitativa de datos de naturaleza cualitativa, tales como los indicadores de aprendizaje representacional, conceptual y proposicional encontrados en los reportes parciales de investigación, es consistente con lo planteado por Miles y Huberman (1994), quienes generaron valores numéricos a partir de datos cualitativos tomando la Universidad Pedagógica Nacional Francisco Morazán, de Honduras, se comparten bajo términos de la Licencia Creative Commons: Se permite que otros puedan descargar las obras y compartirlas con otras personas, siempre y
cuando se reconozca su autoría, pero no se pueden cambiar de ninguna manera ni se pueden utilizer comercialmente. 
como base la identificación de patrones y temas recurrentes, identificación de plausibilidad, identificación de coherencia conceptual y teórica, identificación de construcción de cadenas lógicas de evidencia, agrupamiento de criterios, contabilizaciones, contrastación y comparación y subsumir lo particular en lo general.

Además de generar una tabla de puntajes a partir de la cantidad de indicadores de aprendizaje representacional, conceptual y proposicional encontrados en los 8 reportes, se consideró también relevante evaluar la magnitud de la relación entre los puntajes obtenidos y la cantidad de miembros en cada equipo participante. Para ello, se realizó un análisis de correlación de Spearman que consideró ambas variables. Adicionalmente, se evaluó la magnitud de la relación entre los puntajes obtenidos y los valores de calidad de los reportes de investigación en función de su congruencia con la indagación científica, para lo cual se realizó un nuevo análisis de correlación de Spearman que consideró ambas variables.

\section{Resultados}

\section{Resultados del análisis de datos de calidad del proceso investigativo "en relevo"}

La prueba $\mathbf{T}$ de Student para las calificaciones de calidad asignadas por el revisor 1 y la revisora 2, realizada con un nivel de significancia del 5\% $(\alpha=0.05)$ para muestras emparejadas, mostró un valor de $\mathbf{P}$ de 0.00210588 ( $\mathrm{P}<\alpha$; pues $0.00210588<0.05)$, con un coeficiente de correlación de Pearson de 0.84, descartando de ésta manera que las medias de las calificaciones fueran significativamente iguales, por lo que se determinó utilizar como indicadores para la evaluación de la calidad de los reportes de investigación los promedios entre las calificaciones asignadas por el revisor 1 y la revisora 2, eliminando de esta manera los extremos de ambas mediciones.

La Tabla 1 muestra que un 50\% de los equipos participantes en la etapa de definición del objeto de estudio mostró valores por encima del límite de calidad satisfactoria. El equipo $\mathrm{N}^{\circ} 5$, sin embargo, mostró valores muy cercanos, pero insuficientes para alcanzar el mínimo de calidad esperado.

<Paradigma> - Revista de Investigación Educativa. Año 24. No. 37 
De los 8 equipos participantes, un 75\% no estuvo involucrado en definir el objeto de estudio, lo cual, aunque resulta coherente con la lógica y secuencia de los procesos de investigación, que presentan normalmente el planteamiento de objetivos y variables de investigación como una de sus primeras etapas, refleja para esta modalidad de investigación alternativa la necesidad de hacer énfasis, en equipos que releven de grupos anteriores los objetivos y variables del estudio, en la posibilidad de revisar o replantear los objetivos y variables, con el fin de no desvincular de los elementos fundamentales del problema de estudio a grupos posteriores del proceso de investigación en relevo.

Un $88 \%$ de los equipos (7 equipos) muestran en la Tabla 1 valores de calidad en la revisión de la literatura muy por encima del límite de satisfacción y un $12 \%$ ( 1 equipo) valores inferiores.

Asimismo, la reducida movilidad de los valores de desempeño en esta dimensión, que excluyendo los valores atípicos en los equipos 3 y 7 muestran una desviación estándar de apenas $1.28 \%$ entre los 5 valores restantes, representa un aprovechamiento uniforme de las etapas asociadas con la revisión bibliográfica sobre el problema de estudio entre los equipos participantes.

Resulta especialmente importante considerar el hecho de que el 100\% de los equipos participantes en la implementación piloto tiene a su cargo la función de revisar bibliografía pertinente. Lo anterior representa una de las principales ganancias de la metodología propuesta, y reafirma que adicionalmente a exponer a los estudiantes a etapas y metodologías de investigación a través de su modalidad en relevo, los estudiantes profundizan en un tema de estudio a través del proceso de recepción propuesto por Ausubel (1960, citada por Díaz, 2004), esto es, la utilización de contenidos presentados en su forma final.

Teniendo en cuenta lo anterior resulta importante señalar que una de las funciones de la figura coordinadora del proceso, es sugerir bibliografía de calidad en cada etapa, así como fomentar la revisión profunda de la información relevada por equipos previos a los equipos posteriores, inclusive en etapas ajenas a la construcción de teoría de soporte, lo cual por otro lado podría fortalecer el enlace de la investigación con teoría relevante como principio básico de la indagación científica. la Universidad Pedagógica Nacional Francisco Morazán, de Honduras, se comparten bajo términos de la Licencia Creative Commons: Se permite que otros puedan descargar las obras y compartirlas con otras personas, siempre y cuando se reconozca su autoría, pero no se pueden cambiar de ninguna manera ni se pueden utilizer comercialmente. 
Un $38 \%$ de los equipos participantes (3 equipos) presentaron en calidad en las fuentes de información, valores por encima del mínimo de calidad satisfactoria. E1 62\% restante (5 equipos) presentó valores inferiores, con la mayoría de equipos muy por debajo del valor mínimo esperado.

Lo anterior es un resultado sumamente revelador al considerar la vinculación que éste presenta con el principio de investigación científica "enlace de la investigación con teoría relevante" presentado por Shavelson y Towne (2002).

Del análisis de esta dimensión se observa que además de estimular la consulta de una mayor variedad de documentos, es fundamental promover la revisión de las fuentes en función de su rigor científico, actualidad y reputación. Los bajos valores de desempeño obtenidos por los equipos de investigación se debieron a que los reportes de investigación frecuentemente recurrieron a sitios web y publicaciones no indexadas como fuentes de información en secciones teóricas y metodológicas, lo que representa una importante amenaza a la funcionalidad de la metodología propuesta en el sentido de asentar aspectos teóricos y metodológicos sobre bases escasamente confiables.

Para la dimensión definición de la metodología, un 25\% del total de equipos participantes (2 equipos) presentaron valores superiores al mínimo valor de calidad satisfactoria. El 75\% restante (6 equipos) presentó valores 15 puntos por debajo de lo mínimo necesario.

Esta dimensión, que está altamente relacionada con el principio de "empleo de métodos que permiten la investigación directa de la preguntas de investigación" propuesto por Shavelson y Towne (2002), al analizarse en función de sus indicadores en la Tabla 1, muestra que únicamente un equipo contó entre sus funciones con la responsabilidad de plantear el diseño de la investigación, el cual obtuvo una alta calificación promedio (7 de 8 puntos posibles).

El bajo desempeño en la dimensión, tomando en cuenta que la gran mayoría de equipos no fueron evaluados en función de los dos primeros indicadores de la variable ("Diseño de investigación" y "Población y muestra") por motivo de estar fuera de las funciones asignadas, está atribuido a la calificación obtenida en el tercer indicador (Fase de instrumentación) que presentó valores por debajo del límite aceptable.

<Paradigma> - Revista de Investigación Educativa. Año 24. No. 37 
El indicador "Fase de instrumentación" fue evaluado en función de la validez y confiabilidad comprobada de los instrumentos para recopilar los datos, los cuales, al tratarse de una investigación que requirió la determinación de la concentración de oxígeno disuelto y nutrientes en el agua utilizó para la recopilación de datos un oxinómetro, sistemáticamente calibrado previo a cada medición y de un kit de análisis de nutrientes por colorimetría, el cual aunque fue utilizado de acuerdo a las instrucciones del fabricante, no fue sometido a una comprobación de precisión en los resultados generados.

Teniendo en cuenta este detalle, y considerando que el 50\% de los instrumentos de recolección de datos (1 instrumento) no fue debidamente calibrado, el indicador recibió en ambas revisiones una calificación de 4 puntos de 8 posibles, lo cual, si bien recae sobre las responsabilidades de la coordinación del proceso, repercute negativamente en el desempeño general de la dimensión y del proceso investigativo desarrollado.

Tabla 1

Resultados de desempeño de calidad en los reportes parciales de investigación

\begin{tabular}{|c|c|c|c|c|c|c|c|c|c|}
\hline \multirow[b]{2}{*}{ Dimensiones } & \multirow[b]{2}{*}{ Indicadores } & \multicolumn{8}{|c|}{ Equipos } \\
\hline & & 1 & 2 & 3 & 4 & 5 & 6 & 7 & 8 \\
\hline \multirow{3}{*}{$\begin{array}{c}\text { Calidad en la } \\
\text { definición del } \\
\text { objeto de estudio }\end{array}$} & $\begin{array}{l}\text { Planteamiento de } \\
\text { preguntas de } \\
\text { investigación }\end{array}$ & 7 & $\mathrm{~N} / \mathrm{A}$ & $\mathrm{N} / \mathrm{A}$ & $\mathrm{N} / \mathrm{A}$ & $\mathrm{N} / \mathrm{A}$ & $\mathrm{N} / \mathrm{A}$ & $\mathrm{N} / \mathrm{A}$ & $N / A$ \\
\hline & $\begin{array}{c}\text { Planteamiento de } \\
\text { variables del estudio }\end{array}$ & $\mathrm{N} / \mathrm{A}$ & $\mathrm{N} / \mathrm{A}$ & $\mathrm{N} / \mathrm{A}$ & $\mathrm{N} / \mathrm{A}$ & 7 & $N / A$ & $\mathrm{~N} / \mathrm{A}$ & $N / A$ \\
\hline & $\begin{array}{c}\text { TOTAL } \\
\text { (en porcentaje) }\end{array}$ & 87.5 & 0.0 & 0.0 & 0.0 & 62.5 & 0.0 & 0.0 & 0.0 \\
\hline \multirow{3}{*}{$\begin{array}{l}\text { Calidad en la } \\
\text { revisión de } \\
\text { literatura }\end{array}$} & $\begin{array}{l}\text { Relación de la } \\
\text { revisión de literatura } \\
\text { con el tema de } \\
\text { investigación }\end{array}$ & 6.5 & 6.5 & 8 & 7.5 & 6 & 6.5 & 4 & 6.5 \\
\hline & $\begin{array}{l}\text { Exhaustividad de la } \\
\text { revisión de literatura }\end{array}$ & 7 & 7 & 7 & 6 & 7 & 7 & 5 & 7 \\
\hline & $\begin{array}{c}\text { TOTAL } \\
\text { (en porcentaje) }\end{array}$ & 84.4 & 84.4 & 93.8 & 84.4 & 81.3 & 84.4 & 56.3 & 84.4 \\
\hline
\end{tabular}


Potencial de la investigación "En relevo" para la construcción de saberes y la producción científica

\begin{tabular}{|c|c|c|c|c|c|c|c|c|c|}
\hline \multirow{3}{*}{$\begin{array}{l}\text { Calidad en las } \\
\text { fuentes de } \\
\text { información. }\end{array}$} & $\begin{array}{l}\text { Variedad de las } \\
\text { fuentes de } \\
\text { información }\end{array}$ & 6 & 5 & 4 & 3 & 4 & 2 & 3 & 2 \\
\hline & $\begin{array}{l}\text { Calidad de las } \\
\text { fuentes de } \\
\text { información }\end{array}$ & 7 & 8 & 2.5 & 3.5 & 3.5 & 8 & 2.5 & 8.5 \\
\hline & $\begin{array}{c}\text { TOTAL } \\
\text { (en porcentaje) }\end{array}$ & 81.3 & 81.3 & 40.6 & 40.6 & 46.9 & 62.5 & 34.4 & 65.6 \\
\hline \multirow{4}{*}{$\begin{array}{l}\text { Calidad en la } \\
\text { definición de la } \\
\text { metodología. }\end{array}$} & $\begin{array}{c}\text { Diseño de } \\
\text { investigación }\end{array}$ & $\mathrm{N} / \mathrm{A}$ & $N / A$ & $\mathrm{~N} / \mathrm{A}$ & $\mathrm{N} / \mathrm{A}$ & 7 & $\mathrm{~N} / \mathrm{A}$ & $\mathrm{N} / \mathrm{A}$ & $N / A$ \\
\hline & Población y muestra & $N / A$ & $N / A$ & $N / A$ & $N / A$ & $\mathrm{~N} / \mathrm{A}$ & $N / A$ & $N / A$ & $N / A$ \\
\hline & $\begin{array}{c}\text { Fase de } \\
\text { instrumentación }\end{array}$ & 6 & 4 & 4 & 4 & 4 & 4 & 4 & 4 \\
\hline & $\begin{array}{c}\text { TOTAL } \\
\text { (en porcentaje) }\end{array}$ & 75.0 & 50.0 & 50.0 & 50.0 & 68.8 & 50.0 & 50.0 & 50.0 \\
\hline \multirow{2}{*}{$\begin{array}{l}\text { Calidad en la } \\
\text { descripción de la } \\
\text { recopilación de } \\
\text { datos. }\end{array}$} & $\begin{array}{l}\text { Descripción del } \\
\text { proceso de } \\
\text { recolección de datos }\end{array}$ & 6 & $\mathrm{~N} / \mathrm{A}$ & $\mathrm{N} / \mathrm{A}$ & $N / A$ & $N / A$ & 4 & $\mathrm{~N} / \mathrm{A}$ & $N / A$ \\
\hline & $\begin{array}{c}\text { TOTAL } \\
\text { (en porcentaje) }\end{array}$ & 75.0 & N/A & N/A & N/A & N/A & 50.0 & $\mathrm{~N} / \mathrm{A}$ & N/A \\
\hline \multirow{3}{*}{$\begin{array}{l}\text { Calidad en el } \\
\text { análisis de datos. }\end{array}$} & $\begin{array}{c}\text { Descripción del } \\
\text { análisis a llevarse a } \\
\text { cabo }\end{array}$ & $\mathrm{N} / \mathrm{A}$ & $N / A$ & $N / A$ & $\mathrm{~N} / \mathrm{A}$ & $N / A$ & $N / A$ & $\mathrm{~N} / \mathrm{A}$ & 8 \\
\hline & $\begin{array}{l}\text { Forma de presentar } \\
\text { los resultados }\end{array}$ & 8 & 8 & 8 & 8 & 8 & 8 & 8 & 8 \\
\hline & $\begin{array}{c}\text { TOTAL } \\
\text { (en porcentaje) }\end{array}$ & 100 & 100 & 100 & 100 & 100 & 100 & 100 & 100 \\
\hline \multirow{4}{*}{$\begin{array}{l}\text { Calidad en las } \\
\text { conclusiones del } \\
\text { estudio. }\end{array}$} & $\begin{array}{l}\text { Respuesta a las } \\
\text { preguntas de } \\
\text { investigación }\end{array}$ & $\mathrm{N} / \mathrm{A} \mid$ & $N / A$ & $N / A$ & $\mathrm{~N} / \mathrm{A}$ & $\mathrm{N} / \mathrm{A}$ & $\mathrm{N} / \mathrm{A}$ & $\mathrm{N} / \mathrm{A}$ & 8 \\
\hline & $\begin{array}{l}\text { Análisis crítico de } \\
\text { los resultados }\end{array}$ & $N / A$ & $N / A$ & $N / A$ & $\mathrm{~N} / \mathrm{A}$ & $N / A$ & $N / A$ & $N / A$ & 8 \\
\hline & $\begin{array}{l}\text { Incorporación de la } \\
\text { revisión de literatura }\end{array}$ & $\mathrm{N} / \mathrm{A}$ & $N / A$ & $N / A$ & $\mathrm{~N} / \mathrm{A}$ & $N / A$ & $N / A$ & $N / A$ & 4 \\
\hline & $\begin{array}{c}\text { TOTAL } \\
\text { (en porcentaje) }\end{array}$ & 0.0 & 0.0 & 0.0 & 0.0 & 0.0 & 0.0 & 0.0 & 83.3 \\
\hline \multirow{4}{*}{$\begin{array}{l}\text { Calidad en } \\
\text { referencias y } \\
\text { estilo. }\end{array}$} & Formato y estilo & 5.5 & 4 & 5.5 & 5.5 & 5.5 & 5 & 2 & 6 \\
\hline & $\begin{array}{l}\text { Referencias en el } \\
\text { texto }\end{array}$ & 6 & 6 & 7 & 2 & 6 & $\mathrm{~N} / \mathrm{A}$ & 1.5 & $N / A$ \\
\hline & Bibliografía & 6 & 6 & 7 & 2 & 6 & $N / A$ & 1.5 & $N / A$ \\
\hline & $\begin{array}{c}\text { TOTAL } \\
\text { (en porcentaje) }\end{array}$ & 72.9 & 70.8 & 81.3 & 39.6 & 56.4 & 62.5 & 20.8 & 75.0 \\
\hline
\end{tabular}


La calidad en la descripción de la recopilación de datos, analizada a partir de la exactitud con que fue detallado narrativamente el proceso de recolección de datos, mostró en un $50 \%$ de los equipos (1 equipo de los dos que se vieron involucrados en la etapa), una calificación satisfactoria y un $50 \%$ restante con 15 puntos por debajo del mínimo de calidad establecido. El bajo desempeño en el segundo equipo se debe principalmente a una marcada superficialidad en la descripción de los procesos desarrollados por los equipos.

El $100 \%$ de los equipos participantes se vieron vinculados con la dimensión de Calidad en el análisis de datos, presentando un valor promedio de desempeño de $100 \%$, sobradamente por encima de los valores de calidad satisfactoria establecidos. La dimensión fue evaluada en su totalidad, para 7 de 8 equipos, a partir del indicador de "Forma de presentar los resultados", ya que solamente 1 equipo contó entre sus funciones con la "descripción del análisis a llevarse a cabo".

El indicador "Forma de presentar los resultados" consistió principalmente en evaluar las características de claridad y orden con que los datos recolectados fueron mostrados en cada reporte de investigación parcial. Los equipos participantes, si bien no presentaron resultados finales del análisis de datos en cada etapa de la investigación, sí detallaron en cada reporte los valores de oxígeno y nutrientes determinados en el sitio y en laboratorio respectivamente, lo que fue considerado para fines del presente estudio como resultados parciales del proceso.

Esta dimensión, considerando los indicadores de los cuales se compone, guarda una relación importante con los principios de indagación científica "replica y generaliza entre estudios" y "revela la investigación realizada para estimular la crítica y el escrutinio profesional" propuestos por Shavelson y Towne (2002), en el sentido de que muestran el detalle de procedimientos y la lógica empleada para llegar a resultados, así como la claridad de presentación de éstos, lo cual posibilita el escrutinio profesional por un lado y la réplica de la metodología por estudios posteriores o independientes.

La dimensión de Calidad en las conclusiones del estudio, evaluada exclusivamente para el equipo final del proceso secuencial de investigación en relevo presentó un desempeño de $83.33 \%$ (ver Tabla 1), muy superior al mínimo de calidad establecido. La dimensión fue la Universidad Pedagógica Nacional Francisco Morazán, de Honduras, se comparten bajo términos de la Licencia Creative Commons: Se permite que otros puedan descargar las obras y compartirlas con otras personas, siempre y
cuando se reconozca su autoría, pero no se pueden cambiar de ninguna manera ni se pueden utilizer comercialmente. 
evaluada a través de indicadores relacionados con la coherencia lograda entre las conclusiones y el planteamiento general del objeto de estudio, así como con la teoría de soporte. Por otro lado se analizaron características que demuestran procesos de análisis crítico en la construcción de conclusiones.

Tales indicadores presentan una vinculación importante con 3 principios de indagación científica, a saber: "demuestra una cadena de razonamiento coherente y explícita", "replica y generaliza entre estudios" y "revela la investigación realizada a modo de estimular la crítica y el escrutinio profesional". Valores de desempeño por encima de la calidad satisfactoria representan entonces una prevalencia de principios que en palabras de Shavelson y Towne (2002) son un marco referencial para la realización de inferencias científicas.

Resulta relevante considerar que aunque un $87.5 \%$ de los equipos participantes no cuenta entre sus funciones con la definición de conclusiones finales, teniendo en cuenta la lógica y el funcionamiento normal de los procesos de investigación científica, dimensiones como la de calidad en el análisis de datos evidencian que en efecto existen a lo largo del proceso investigativo resultados parciales que individualmente no generan conclusiones pero que implican la utilización de instrumentos de recolección de datos para producir resultados parciales que eventualmente serán procesados para dar respuestas a hipótesis o preguntas de investigación a través de lo que Ausubel (1960, citada por Díaz, 1999) denomina descubrimiento, marcado por el logro de conocimiento a través de la experiencia directa.

Con respecto a la "Calidad en referencias y estilo", un 50\% de los equipos participantes (4 equipos) mostraron valores por encima del nivel de calidad satisfactorio establecido. El 50\% restante presentó diferentes niveles de desempeño por debajo del mínimo de calidad.

Tal dimensión fue evaluada en función de indicadores de practicidad y estética en la documentación del proceso investigativo así como en la calidad del proceso de referenciación, fundamental para la construcción científica y la vinculación entre el trabajo construido por equipos de investigadores.

Los bajos valores de desempeño en la dimensión son producto de una evidente falta de familiaridad en los estudiantes de los equipos

<Paradigma> - Revista de Investigación Educativa. Año 24. No. 37 
participantes con los métodos de referenciación y de presentación de bibliografía consultada. De lo anterior resulta evidente que una de las principales funciones de la figura de coordinación del proceso de investigación en relevo deberá centrarse en visibilizar la relevancia de una adecuada referenciación de fuentes revisadas.

Resultados del análisis de la prevalencia de procesos potenciadores del aprendizaje investigativo

El 100\% de los equipos muestra indicadores de procesos asociados a la construcción de aprendizaje significativo representacional, conceptual y proposicional; sin embargo, los totales ponderados presentados en la Tabla 2, presentan un valor promedio de 44.25 puntos y una desviación estándar de 48.30 puntos entre los equipos participantes. De lo anterior, puede distinguirse la presencia de valores extremos de hasta más de tres desviaciones estándar, lo que indica una alta variabilidad en los datos obtenidos y que por ende permite inferir un distinto grado de prevalencia de procesos potenciadores de aprendizaje significativo entre los equipos participantes del ensayo piloto tal como puede observarse en la Gráfica 1.

\section{Gráfica 1}

Indicadores de procesos asociados a la construcción de aprendizaje representacional, conceptual y proposicional

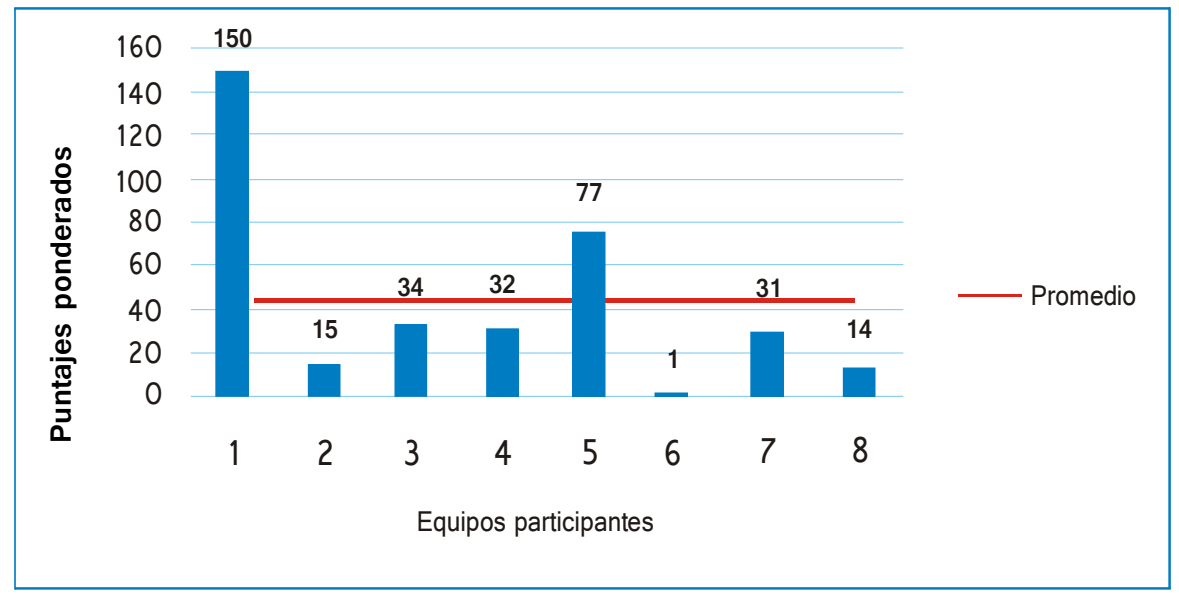

Del análisis del recuento desagregado de indicadores presentado en la Gráfica 2 se identifica una mayor prevalencia procesos asociados a la construcción de aprendizaje conceptual, con un $75 \%$ de los reportes la Universidad Pedagógica Nacional Francisco Morazán, de Honduras, se comparten bajo términos de la Licencia Creative Commons: Se permite que otros puedan descargar las obras y compartirlas con otras personas, siempre y
cuando se reconozca su autoría, pero no se pueden cambiar de ninguna manera ni se pueden utilizer comercialmente. 
parciales de investigación mostrando una marcada superioridad en el recuento de evidencias de procesos asociados a este tipo de aprendizaje con respecto al recuento de evidencias de procesos asociados a la construcción de aprendizaje representacional y proposicional.

\section{Gráfica 2}

Recuento de evidencias de procesos asociados a la construcción de aprendizaje representacional, conceptual y proposicional

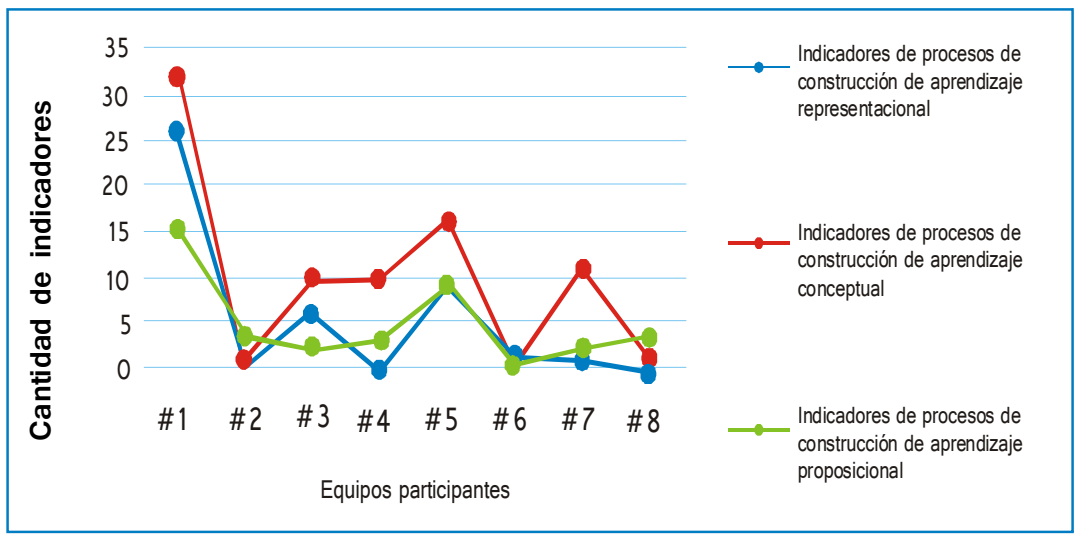

Se realizó un recuento de procesos asociados a la construcción de aprendizaje significativo por cada sección del documento en los dos reportes con valores sobresalientes, observándose que de 150 puntos alcanzados por el equipo \#1 en la ponderación de indicadores presentes en el reporte de investigación, 116 (77\%) están concentrados en la sección de Marco Teórico.

Con respecto al equipo \#5, de 77 puntos alcanzados en la ponderación de indicadores presentes en el reporte de investigación, 47 (61\%) están concentrados en la sección de Marco Metodológico.

\section{Tabla 2}

Recuento y ponderación de indicadores de procesos asociados a la construcción de aprendizaje representacional, conceptual y proposicional

\begin{tabular}{|c|c|c|c|c|c|c|c|c|}
\hline \multirow[b]{2}{*}{ Recuento de indicadores } & \multicolumn{8}{|c|}{ Informe parcial de investigación } \\
\hline & $\# 1$ & \#2 & \#3 & \#4 & \#5 & \#6 & \#7 & \#8 \\
\hline $\begin{array}{l}\text { Indicadores de procesos de construcción de } \\
\text { aprendizaje representacional }\end{array}$ & 26 & 1 & 6 & 0 & 9 & 1 & 1 & 0 \\
\hline
\end{tabular}

<Paradigma> - Revista de Investigación Educativa. Año 24. No. 37 


\begin{tabular}{|c|c|c|c|c|c|c|c|c|}
\hline $\begin{array}{l}\text { Indicadores de procesos de construcción de } \\
\text { aprendizaje conceptual }\end{array}$ & 32 & 1 & 10 & 10 & 16 & 0 & 11 & 1 \\
\hline $\begin{array}{l}\text { Indicadores de procesos de construcción de } \\
\text { aprendizaje proposicional }\end{array}$ & 15 & 3 & 2 & 3 & 9 & 0 & 2 & 3 \\
\hline Ponderación de indicadores & $\# 1$ & $\# 2$ & \#3 & \#4 & \#5 & \#6 & $\# 7$ & \#8 \\
\hline $\begin{array}{l}\text { Cantidad de indicadores de aprendizaje } \\
\text { representacional } \times 1.00\end{array}$ & 26 & 1 & 6 & 0 & 9 & 1 & 1 & 0 \\
\hline $\begin{array}{l}\text { Cantidad de indicadores de aprendizaje } \\
\text { conceptual } \times 2.00\end{array}$ & 64 & 2 & 20 & 20 & 32 & 0 & 22 & 2 \\
\hline $\begin{array}{l}\text { Cantidad de indicadores de aprendizaje } \\
\text { proposicional } \times 4.00\end{array}$ & 60 & 12 & 8 & 12 & 36 & 0 & 8 & 12 \\
\hline Totales ponderados & 150 & 15 & 34 & 32 & 77 & 1 & 31 & 14 \\
\hline
\end{tabular}

Además del recuento de indicadores por sección de cada reporte, se condujo un análisis de correlación de Spearman cuyos resultados reflejaron que ni el tamaño de los equipos participantes, ni la calificación obtenida en el análisis de calidad de los reportes parciales de investigación guardan una correlación con la prevalencia de procesos asociados a la construcción de aprendizaje significativo representacional, conceptual o proposicional $(\mathrm{P}=0.468>\alpha=0.05$ con valor de Rho de Spearman de 0.301 y $\mathrm{P}=0.867>\alpha=0.05$ con un valor de Rho de Spearman de 0.07, respectivamente).

\section{Conclusiones}

La metodología propuesta, aunque mostró en su implementación piloto altos grados de variabilidad intergrupal, parece presentar mejores posibilidades de exponer de manera más homogénea a los principios de indagación científica y construcción de aprendizaje significativo al implementarse para desarrollar proyectos investigativos del tipo longitudinal, en el cual cada uno de los equipos participantes, además de contar con funciones específicas en su participación en el proceso esté necesariamente involucrado en la colecta de datos, pues relegar tal función a una menor cantidad de grupos incorporaría otro factor más de variabilidad en materia de desempeño en calidad y de prevalencia de indicadores de aprendizaje, y consecuentemente, mayor variabilidad en el aprovechamiento de la experiencia.

A pesar de que no existe una correlación estadística entre la calidad de los reportes parciales de investigación y las evidencias de procesos la Universidad Pedagógica Nacional Francisco Morazán, de Honduras, se comparten bajo términos de la Licencia Creative Commons: Se permite que otros puedan descargar las obras y compartirlas con otras personas, siempre y cuando se reconozca su autoría, pero no se pueden cambiar de ninguna manera ni se pueden utilizer comercialmente. 
asociados a la construcción de aprendizaje significativo, el desempeño de ambas variables mostró recurrentemente tendencias positivas asociadas al involucramiento de los equipos participantes en funciones específicas como la definición del objeto de estudio y la construcción de Marco Teórico y Metodológico.

Lo anterior implica que nuevas experiencias de implementación de la metodología propuesta deberían tomar en cuenta la importancia de fomentar la participación activa y permanente de todos los equipos en etapas de esa índole, así como reforzar la idea de que realizar enmiendas a procesos o resultados alcanzados por equipos previos es una práctica deseable que estaría posibilitando un mayor grado de exposición a etapas potencialmente generadoras de aprendizaje significativo y de internalización de principios de indagación científica.

Puntos de control de calidad como la falta de calibración rigurosa del equipo a utilizar imposibilitó el uso de los resultados obtenidos en el ensayo piloto como valores fidedignos para análisis del tema estudiado, por lo que es recomendable que la figura de coordinación del proyecto de investigación en relevo asuma un mayor grado de control sobre variables de este tipo, y no exclusivamente sobre la dosificación y programación de las actividades a cargo de los estudiantes, con la finalidad de maximizar el verdadero potencial de prácticas de esta naturaleza para la producción científica.

En este sentido resultaría también conveniente evaluar esquemas alternativos para la implementación de la investigación en relevo, tales como definir un proyecto investigativo de mayor profundidad que se abra a la participación de estudiantes con diferentes grados de involucramiento dependiendo de su estado de avance en el plan de estudios, o bien la implementación piloto de nuevos proyectos de investigación en relevo con cursos significativamente más avanzados que permitan contrastar el desempeño obtenido en el presente estudio.

La aplicación de rúbricas especializadas en cuantificar el desempeño del equipo de investigadores en función de rasgos deseables en los procesos generales de investigación, permite una medida uniforme para evaluar el desempeño y potencial de la investigación en relevo a diferentes niveles educativos y áreas del conocimiento, provocar reformas metodológicas a su implementación y evaluarla como herramienta para la potenciación de competencias investigativas concretas. 


\section{Referencias Bibliográficas}

Alonso, M. (2010). Variables del aprendizaje significativo para el desarrollo de las competencias básicas. Tenerife: Prácticas del profesorado.

Ausubel, D. (1983). Psicología educativa: Un punto de vista cognoscitivo. México,D.F.: Trillas.

Ausubel, D. (2000). The acquisition and retention of knowledge: A cognitive view. Philadeplhia: Springer Science \& Business Media.

Díaz, F; Hernández, G. (2004). Estrategias docentes para un aprendizaje significativo, una interpretación constructivista. México, D.F.: MCGraw Hill.

Miles, M; Huberman, A. (1994). An expanded sourcebook: Data Analysis. Londres: Sage Publications.

Novak, J. (2011). Meaningful learning underlies the constructive integration of thinking, feeling and acting. Aprendizag em Significativa em Revista/Meaningful Learning Review, 1(2), 1-14.

Shavelson, R. J., \& Towne, L. (2002). Scientific research in education. Committee on Scientific Principles for Education Research. Center for Education. Division of Behavioral and Social Sciences and Education. National Research Council. Washington, DC: National Academy Press.

Universidad de Puerto Rico. (2011). Rúbrica para trabajos de Investigación. Recuperado del sitio web del banco de rúbricas de la Universidad de Puerto Rico, recinto de Río Piedras Escuela Graduada de Ciencias y Tecnologías de la Información, de http://egcti.upr.edu/ index.php/2012-11-15-15-14-28/rubricas

Universidad Pedagógica Nacional Francisco Morazán. (2011). Observatorio Universitario de la Educación Nacional. Recuperado del sitio web del Instituto de Investigación y Evaluación Educativa de la Universidad Pedagógica Nacional Francisco Morazán, de http:// postgrado.upnfm.edu.hn/paradigma/revistas/boletines/ observatorio $\% 209 \% 20 \mathrm{enemz} \% 202011$.pdf 